\title{
Design and measurement of a W-band Brewster window
}

\author{
Yanyan Zhang, Liang Zhang, Wenlong He, Craig R. Donaldson, Paul McElhinney, Alan D. R. Phelps \\ and Adrian W. Cross
}

\begin{abstract}
A Brewster window integrated into a corrugated waveguide was designed, manufactured and studied for a W-band gyrotron traveling wave amplifier. A corrugated waveguide intended to guide a quasi-plane wave $\left(\mathrm{HE}_{11}\right)$ through the window, was numerically optimized and verified by measurement using a Vector Network Analyzer. The Brewster window was measured to have a reflection lower than $-22 \mathrm{~dB}$ for the $\mathrm{TE}_{11}$ mode over the frequency range of $85-101 \mathrm{GHz}$.
\end{abstract}

Index Terms - Brewster window, Gyro-devices, corrugated waveguide.

\section{INTRODUCTION}

The gyrotron traveling wave amplifier (gyro-TWA) has attracted huge research interest in the past decades for a variety of applications including high resolution radar, high frequency communications, plasma diagnostics, materials processing etc. [1], [2]. A W-band gyro-TWA based on a helically corrugated waveguide [3], [4] and a cusp electron gun is currently being studied to achieve an output power of $5 \mathrm{~kW}$ in the frequency band of $90-100 \mathrm{GHz}$. To successfully operate such a wide bandwidth amplifier, an output microwave window of sufficient low reflection in the operating frequency range is required.

The functions of the window are to separate the high vacuum inside the electronic device from the outside atmospheric environment, and to couple out microwave power at the same time. The ideal window should have low power absorption, high transmission, high mechanical strength, high thermal conductivity and have vacuum integrity to the required level. Different types of windows have been developed, such as single-disc, multi-disc, pillbox and Brewster windows, for different applications. The single-disc window [5] has excellent

Manuscript received June 16, 2015; revised August 06, 2015, August 20, 2015; accepted August 20, 2015. This work was supported by the Engineering and Physical Sciences Research Council (EPSRC) U.K. under Research Grant EP/K029746/1. The EPSRC Engineering Instrument Pool is gratefully acknowledged for providing the Vector Network Analyzer (VNA) used in the experiments. Y. Zhang would like to thank the China Scholarship Council for its financial support.

The authors are with the Department of Physics, SUPA, University of Strathclyde, Glasgow, G4 0NG, Scotland, UK (email: zhangyanyanzyy @ hotmail.com, liang.zhang@strath.ac.uk,w.he@ strath.ac.uk, craig.donaldson @ strath.ac.uk, paul.mcelhinney@strath.ac.uk, a.d.r.phelps@strath.ac.uk, a.w.cross@strath.ac.uk).

Y. Zhang is also with the Terahertz Science and Technology Research Center, School of Physical Electronics, University of Electronic Science and Technology of China, Chengdu 610054, China. transmission over a narrow frequency band $(\sim 1 \%)$. The narrow bandwidth is mainly caused by the impedance mismatch between the two waveguide media. This mismatch could be compensated by introducing additional impedance matching sections, through either changing the waveguide geometries or adding dielectric discs of suitable dielectric constants, as occurs in the pillbox [6] and multi-disc [7] types of microwave window. However the sensitivity to the geometrical tolerances of these windows will limit the bandwidth performance when operating at high frequencies.

The Brewster window has characteristically broad bandwidth. The reflections of incident linearly polarized waves would be vanishingly small and independent of frequency if the wave enters at the Brewster angle. The Brewster window, although not being a new concept, has not been widely published. However it has been successfully used in step tunable gyrotrons at $140 \mathrm{GHz}$ and $170 \mathrm{GHz}$ [8]. In this paper, simulation and the measurement of a W-band Brewster window in a corrugated waveguide is presented.

\section{THEORY OF THE BREWSTER WINDOW}

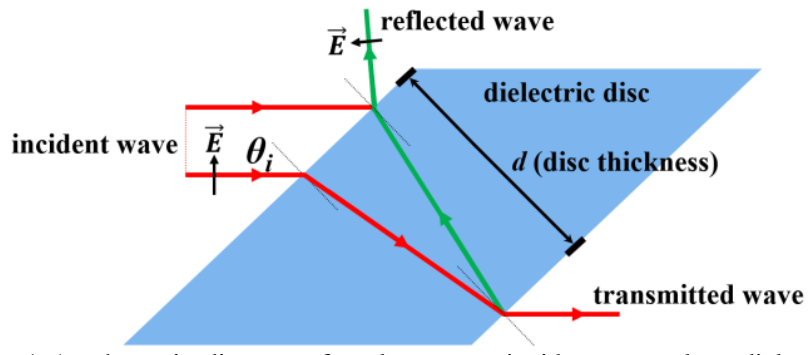

Fig. 1 A schematic diagram of a plane wave incident on a plane dielectric boundary.

Fig. 1 shows the schematic diagram of the wave reflection and refraction in the single dielectric disc, where $d$ is the thickness of the dielectric disc. For the wave with polarization in the plane of incidence, the Brewster angle is defined by the incident angle $\theta_{i}$ with zero reflection. It follows the Brewster's law and is equal to $\theta_{\text {Brew }}=\arctan \left(\sqrt{\varepsilon_{r 2}^{\prime} / \varepsilon_{r 1}^{\prime}}\right)$, where $\varepsilon_{r 1}^{\prime}$ and $\varepsilon_{r 2}^{\prime}$ represent the relative permittivity of the medium in which the incident wave is propagating and that of the dielectric disc respectively. Similarly in the case of an incident wave with its plane of polarization perpendicular to the plane of incidence, the reflection can vanish if the phase displacement of the first reflection and the wave scattered out of the material is a multiple of $(2 n+1) \pi$. The relationship between the incident 
angle and the thickness of the dielectric material $d$ with vacuum as the background media $\left(\varepsilon_{r 1}^{\prime}=1\right)$ can be derived based on Fig. 1 and it follows that

$\sin \theta_{i}=\sqrt{\varepsilon_{r 2}^{\prime}\left[1-\left(N \frac{\lambda_{n} / 2}{d}\right)^{2}\right]}, N \in\left(\frac{d}{\lambda_{n} / 2} \sqrt{\frac{\varepsilon_{r 2}^{\prime}-1}{\varepsilon_{r 2}^{\prime}}}, \frac{d}{\lambda_{n} / 2}\right), n=1,2,3 \ldots$

where $N$ is an integer and $\lambda_{n}$ is the wavelength of the incident wave in the dielectric disc. If the reflections of both the perpendicular and the parallel polarizations are required to vanish for at least one frequency, then the optimal thickness of the Brewster window $d_{N}$ needs to be chosen according to

$$
d_{N}=N \frac{\lambda_{n}}{2} \sqrt{\frac{\varepsilon_{r 2}^{\prime}+1}{\varepsilon_{r 2}^{\prime}}}=N \frac{c}{2 f} \frac{\sqrt{\varepsilon_{r 2}^{\prime}+1}}{\varepsilon_{r 2}^{\prime}}
$$

where $c$ is the speed of light, $f$ is the desired frequency that has 'zero' reflection [9]. The Brewster window also has low insertion loss as the incident wave only passes through the window material once.

In this paper, quartz was chosen as the window material and $N=1$ was used in the simulation and manufacture. With a dielectric constant of 3.75 and a center frequency of $95 \mathrm{GHz}$, the Brewster angle and thickness of the quartz disc are $62.7^{\circ}$ and $0.92 \mathrm{~mm}$, respectively. Thicker material $(N>1)$ could also be used to increase the mechanical strength and to facilitate vacuum brazing.

\section{SimUlation OF THE BREWSTER WINDOW}

As the wave mode after the interaction region of the $\mathrm{W}$-band gyro-TWA is the circular $\mathrm{TE}_{11}$ mode and the Brewster window requires a plane wave, a corrugated horn that converts the $\mathrm{TE}_{11}$ mode into the Gaussian-like $\mathrm{HE}_{11}$ mode, a quasi-plane wave, was therefore used [10]. The measurement showed that the corrugated horn had low reflection of around $-30 \mathrm{~dB}$ and a Gaussian percentage of $98 \%$ [11]. Such a mode can be composed by combining approximately $88.1 \%$ of a $\mathrm{TE}_{11}$ mode with $11.9 \%$ of a $\mathrm{TM}_{11}$ mode with a relative phase difference of $180^{\circ}$ between the two modes and by neglecting higher TE/TM modes.

To maintain the Gaussian-like $\mathrm{HE}_{11}$ mode, a corrugated waveguide (Fig. 2) with radius of $12.6 \mathrm{~mm}$ was optimized to accommodate the Brewster window [12]. The scattering parameters and mode contents of such a corrugated waveguide can be efficiently calculated by a mode-matching method [13]. An in-house numerical code in MATLAB was used to optimize the corrugation depth $h$, the vane lengths $d_{1}$ and gap $d_{2}$. The goal function in the optimization is to minimize the differences between the input and output hybrid $\mathrm{TE}_{11}$ and $\mathrm{TM}_{11}$ mode content over the frequency band of $90-100 \mathrm{GHz}$. The initial geometry parameters are chosen as the same as the last section of the corrugated horn. The optimal geometry was $h=0.87 \mathrm{~mm}$, $d_{1}=0.72 \mathrm{~mm}$ and $d_{2}=0.77 \mathrm{~mm}$ and with a total period number of 64 . The mode content in this corrugated waveguide is $87.4 \% \mathrm{TE}_{11}, 11.6 \% \mathrm{TM}_{11}$ with relative phase difference of $180^{\circ}$. The performance of the corrugated waveguide was verified by using the 3D FDTD code CST Microwave Studio. Sufficiently dense meshes were used to limit the numerical error to a negligible level. The mode contents of the $\mathrm{TE}_{11}$ and $\mathrm{TM}_{11}$ modes at the input and output port were confirmed to be the same and the reflections for both the $\mathrm{TE}_{11}$ and $\mathrm{TM}_{11}$ modes were below $-30 \mathrm{~dB}$.

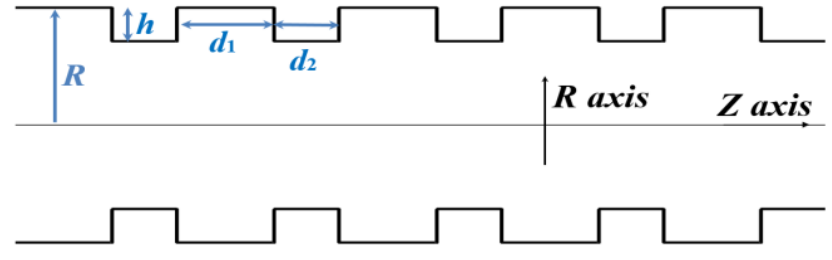

Fig. 2 The structure of the corrugated waveguide.

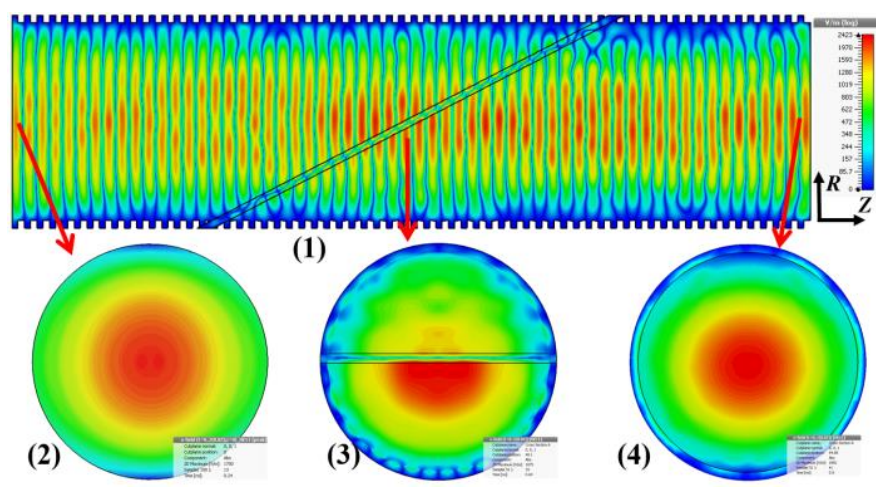

(a)

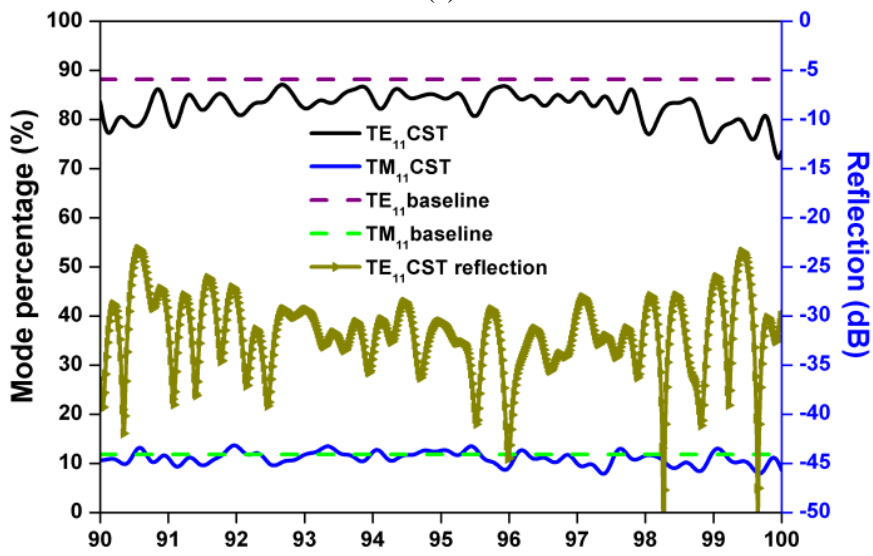

(b)

Fig. 3 (a) The electric field at 95 GHz: (1) $x$-z plane where $y=0$; (2) $x-y$ plane where $z=0$ (3) $x-y$ plane at the center of the window; (4) $x-y$ plane at the end of the window and (b) the simulated mode contents of the $\mathrm{TE}_{11}$ and $\mathrm{TM}_{11}$ modes as well as the reflection of the $\mathrm{TE}_{11}$ mode.

The Brewster window was then inserted into the corrugated waveguide in the CST simulation. In this case a $\mathrm{HE}_{11}$ mode resembles 'quasi-optical' propagation and its wavelength is approximately equal to the free space wavelength. The quartz disc with a thickness of $0.92 \mathrm{~mm}$ was initially used and placed at the Brewster angle of $62.7^{\circ}$. A less than $-20 \mathrm{~dB}$ reflection was found for the $\mathrm{TE}_{11}$ mode, which meets the requirement of the W-band gyro-TWA. Further simulations by a parameter sweep found that a slightly thinner disc has bigger reflection at lower frequencies and smaller reflection at higher frequencies. The thickness of $0.90 \mathrm{~mm}$ was able to achieve better performance over the simulated frequency range. Fig. 3(b) shows the simulated mode content of the $\mathrm{TE}_{11}$ and $\mathrm{TM}_{11}$ modes, as well as the reflection of the $\mathrm{TE}_{11}$ mode. A smaller than $-22 \mathrm{~dB}$ reflection of the $\mathrm{TE}_{11}$ mode can be achieved over the whole 
frequency band. This verifies the wide bandwidth of the Brewster window. When reducing the thickness of the window material, the reflection will become larger at lower frequencies and become smaller at the higher frequencies. Increasing the thickness of the window will have the opposite trend. Fig. 3(b) also shows that the mode content of the $\mathrm{TE}_{11}$ mode is a few percent less than the input power, which is mainly caused by the transmission loss within the quartz material. This would result in a slight distortion in the field distribution of the Gaussian-like $\mathrm{HE}_{11}$ mode as it differs from the ideal mode content of the $\mathrm{TE}_{11}$ and $\mathrm{TM}_{11}$ modes. Fig. 3(a) shows that the electric field pattern at the output port of the window has only a slight distortion compared with the one at the entrance. The Gaussian content at the output port was calculated to be $97.7 \%$, compared with $97.9 \%$ with the ideal hybrid mode mixture.

Parameter sweeps were carried out to study the sensitivity of the assembling angle of the window. The tolerance of the assembling angle is about $\pm 1^{\circ}$ in order to keep the reflection lower than $-20 \mathrm{~dB}$ over the whole frequency band from $90-100$ $\mathrm{GHz}$. With an angle $1^{\circ}$ smaller than the designed angle, the reflection at the higher frequency side becomes worse and is larger than $-20 \mathrm{~dB}$, while a larger angle makes the lower frequency worse. The bandwidth is narrower than the theoretical calculation due to the imperfect Gaussian wave inside the corrugated waveguide.

\section{CONSTRUCTION AND MEASUREMENTS}

A prototype of a Brewster window was manufactured and its microwave properties measured to verify the simulations. A corrugated waveguide with optimized dimensions was manufactured using 3D printing technology. The waveguide was then connected to the output of the corrugated horn, to measure its performance using a Vector Network Analyzer (VNA), as shown in Fig. 4. The corrugated waveguide was wire cut and assembled with the quartz disc to form the Brewster window after the measurement. Fig. 5 shows the measured reflection of the corrugated horn with and without the corrugated waveguide. The two traces have very similar reflection levels, showing that the corrugated waveguide does not cause additional reflection. The reflection of the corrugated horn as well as the Brewster window is also shown in Fig. 5. The reflection is less than $-22 \mathrm{~dB}$ over the frequency range of $85-101 \mathrm{GHz}$, which covers the operation frequency range of the gyro-TWA from $90-100 \mathrm{GHz}$.

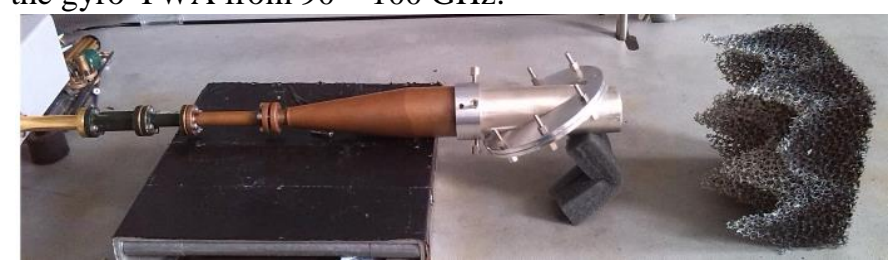

Fig. 4 The assembled Brewster window and the measurement setup.

\section{CONCLUSION}

A W-band Brewster window in a corrugated waveguide has been designed and measured. The Brewster window was measured to have lower than $-22 \mathrm{~dB}$ reflection over the frequency range of $85-101 \mathrm{GHz}$, which satisfies the requirement of the gyro-TWA.

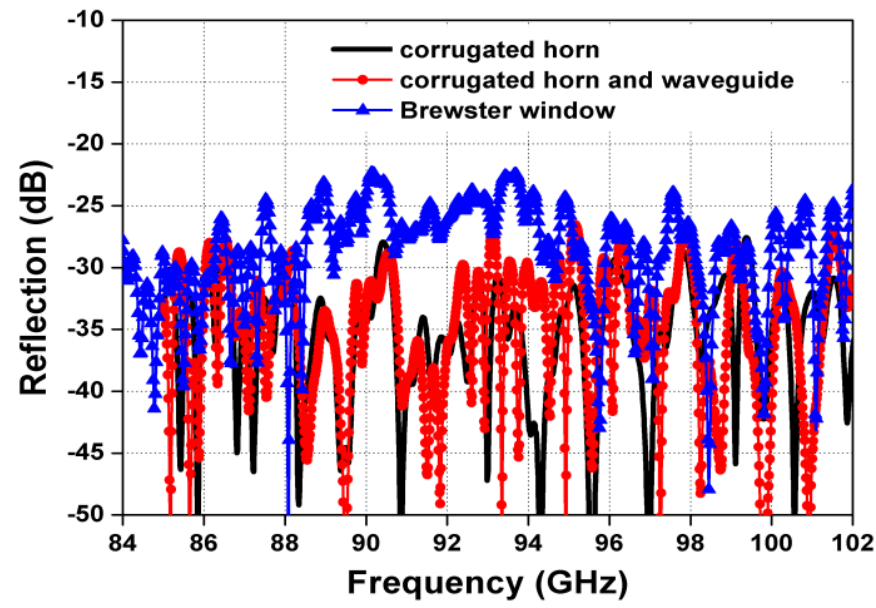

Fig. 5 Measurement of the corrugated horn with and without the corrugated waveguide, as well as the Brewster window.

\section{REFERENCES}

[1] P. Woskoboinikow, "Development of gyrotrons for plasma diagnostics (invited)," Rev. Sci. Instrum., vol. 57, no. 8, pp. 2113-2118, Aug. 1986.

[2] Shoji Miyake, "Millimeter-Wave Materials Processing in Japan by High-Power Gyrotron," IEEE Trans. Plasma Sci, vol. 31, no. 5, pp. 1010-1015, Oct. 2003.

[3] W. He, C. R. Donaldson, L. Zhang, K. Ronald, P. McElhinney and A. W. Cross, "High power wideband gyrotron backward wave oscillator operating towards the terahertz region," Phys. Rev. Lett. vol. 110, no. 16, p.165101, Apr. 2013.

[4] L. Zhang, W. He, K. Ronald, A. D. R. Phelps, C. G. Whyte, C. W. Robertson, A. R. Young, C. R. Donaldson and A. W. Cross, "Multi-mode coupling wave theory for helically corrugated waveguide," IEEE Trans. Microw. Theory Techn., vol. 60, no. 1, pp. 1-7, Jan. 2012.

[5] M. E. Hill, R. S. Callin and D. H. Whittum, "High-Power Vacuum Window in WR10," IEEE Trans. Microw. Theory Techn., vol. 49, no. 5, pp. $994-995$, May 2001

[6] A. M. Cook, C. D. Joye, T. Kimura, E. L. Wright and J. P. Calame, "Broadband 220-GHz Vacuum Window for a Traveling-Wave Tube Amplifier," IEEE Trans. Electron Devices, vol. 60, no. 3, pp. 1257-1259, Mar. 2013.

[7] C. R. Donaldson, W. He, L. Zhang and A. W. Cross, "A W-Band Multi-Layer Microwave Window for Pulsed Operation of Gyro-Devices," IEEE Microwave Wireless Compon. Lett., vol. 23, no. 5, pp. 237 - 239, May 2013.

[8] M. Thumm, A. Arnold, E. Borie, O. Braz, G. Dammertz, O. Dumbrajs, K. Koppenburg, M. Kuntze, G. Michel and B. Piosczyk, "Frequency step-tunable (114-170GHz) megawatt gyrotrons for plasma physics applications," Fusion Eng. Des., vol. 53, pp. 407-421, Jan. 2001.

[9] X. Yang, G. Dammertz, R. Heidinger, K. Koppenburg, F. Leuterer, A. Meier, B. Piosczyk, D. Wagner and M. Thumm, "Design of an ultra-broadband single-disk output window for a frequency step-tunable 1 MW gyrotron,” Fusion Eng. Des., vol. 74, pp. 489-493, Nov. 2005.

[10] P. McElhinney, C. R. Donaldson, L. Zhang and W. He, "A High Directivity Broadband Corrugated Horn for W-band Gyro-devices," IEEE Trans. Antennas Propag., vol. 61, no. 3, pp. 1453-1456, Mar. 2013.

[11] Paul F. Goldsmith, "Quasioptical Systems: Gaussian Beam Quasioptical Propagation and Applications," chap. 6, Wiley-IEEE Press, 1997.

[12] M. Read, T. Bui, D. Marsden, R. L. Ives, B. Stockwell and J. Neilson, "Development of a wide-band window in $\mathrm{HE}_{1,1}$ guide for gyrotrons," IEEE 14th International Vacuum Electronics Conference (IVEC), Paris, France, 21-23 May 2013, pp. 1-2.

[13] T. Itoh, Ed., "Numerical techniques for microwave and millimeter-wave passive structures," chap. 9-10, New York: Wiley, 1989. 